Bull. Korean Math. Soc. 52 (2015), No. 4, pp. 1327-1338

http://dx.doi.org/10.4134/BKMS.2015.52.4.1327

\title{
THE $w$-WEAK GLOBAL DIMENSION OF COMMUTATIVE RINGS
}

\author{
FAngGui WANG AND LeI QIAO
}

\begin{abstract}
In this paper, we introduce and study the $w$-weak global dimension $w$-w.gl.dim $(R)$ of a commutative $\operatorname{ring} R$. As an application, it is shown that an integral domain $R$ is a Prüfer $v$-multiplication domain if and only if $w$-w.gl.dim $(R) \leqslant 1$. We also show that there is a large class of domains in which Hilbert's syzygy Theorem for the $w$-weak global dimension does not hold. Namely, we prove that if $R$ is an integral domain (but not a field) for which the polynomial ring $R[x]$ is $w$-coherent, then $w$-w.gl.dim $(R[x])=w$-w.gl.dim $(R)$.
\end{abstract}

\section{Introduction}

Recall that a Prüfer domain is an integral domain in which every finitely generated ideal is invertible. It is well-known that the concept of Prüfer domains has played a central role in the development of the classical ideal theory. An important generalization of the Prüfer domain notion is that of a Prüfer $v$-multiplication domain (PVMD). This notion comes from multiplicative ideal theory, and various ideal-theoretic properties of it have been considered by many authors, see for example $[1,2,4,7,9,10,13,14,15]$. From the homological algebra point of view, Prüfer domains are exactly the integral domains of weak global dimension at most one. The original motivation for this work is to provide a homological algebra characterization of PVMDs. To do so, we need the notion of a $w$-flat module. Recently, modules of this type have received attention in several papers in the literature, see for example [3, 11, 21, 22].

Throughout, $R$ denotes a commutative ring with an identity element and all modules are unitary.

Now, we review some definitions and notation. Let $J$ be an ideal of $R$. Following [25], $J$ is called a Glaz-Vasconcelos ideal (a $G V$-ideal for short) if $J$ is finitely generated and the natural homomorphism $\varphi: R \rightarrow J^{*}=\operatorname{Hom}_{R}(J, R)$ is an isomorphism. Note that the set $\mathrm{GV}(R)$ of $\mathrm{GV}$-ideals of $R$ is a multiplicative

Received September 8, 2014.

2010 Mathematics Subject Classification. Primary 13D05, 13A15; Secondary 13 F05.

Key words and phrases. GV-torsionfree module, $w$-module, $w$-flat module, $w$-flat dimension, $w$-weak global dimension. 
system of ideals of $R$. Let $M$ be an $R$-module. Define

$$
\operatorname{tor}_{\mathrm{GV}}(M)=\{x \in M \mid J x=0 \text { for some } J \in \mathrm{GV}(R)\} .
$$

Thus $\operatorname{tor}_{\mathrm{GV}}(M)$ is a submodule of $M$. Now $M$ is said to be GV-torsion (resp., $\mathrm{GV}$-torsionfree) if $\operatorname{tor}_{\mathrm{GV}}(M)=M$ (resp., $\left.\operatorname{tor}_{\mathrm{GV}}(M)=0\right)$. A GV-torsionfree module $M$ is called a $w$-module if $\operatorname{Ext}_{R}^{1}(R / J, M)=0$ for any $J \in \mathrm{GV}(R)$. Then projective modules and reflexive modules are both $w$-modules. In the recent paper [26], it was shown that all flat modules are $w$-modules. Let $w$-Max $(R)$ denote the set of $w$-ideals of $R$ maximal among proper integral $w$-ideals of $R$ and we call $\mathfrak{m} \in w$-Max $(R)$ a maximal $w$-ideal of $R$. Then by [25, Proposition 3.8 ] every maximal $w$-ideal is prime. Notice that an $R$-module $M$ is GV-torsion if and only if $M_{\mathfrak{m}}=0$ for all $\mathfrak{m} \in w-\operatorname{Max}(R)$ (see [23, Theorem 2.7]). For any GV-torsionfree module $M$,

$$
M_{w}=\{x \in E(M) \mid J x \subseteq M \text { for some } J \in \mathrm{GV}(R)\}
$$

is a $w$-submodule of $E(M)$ containing $M$ and is called the $w$-envelope of $M$, where $E(M)$ denotes the injective hull (or envelope) of $M$. It is clear that a GV-torsionfree module $M$ is a $w$-module if and only if $M_{w}=M$. Let $M$ and $N$ be $R$-modules and let $f: M \rightarrow N$ be a homomorphism. Following [20], $f$ is called a $w$-monomorphism (resp., w-epimorphism, w-isomorphism) if $f_{\mathfrak{m}}$ : $M_{\mathfrak{m}} \rightarrow N_{\mathfrak{m}}$ is a monomorphism (resp., an epimorphism, an isomorphism) for all $\mathfrak{m} \in w$-Max $(R)$. A sequence $A \rightarrow B \rightarrow C$ of modules and homomorphisms is called $w$-exact if the sequence $A_{\mathfrak{m}} \rightarrow B_{\mathfrak{m}} \rightarrow C_{\mathfrak{m}}$ is exact for all $\mathfrak{m} \in w$-Max $(R)$. An $R$-module $M$ is said to be of finite type if there exists a finitely generated free $R$-module $F$ and a $w$-epimorphism $g: F \rightarrow M$. Similarly, an $R$-module $M$ is said to be of finitely presented type if there exists a $w$-exact sequence $F_{1} \rightarrow F_{0} \rightarrow M \rightarrow 0$, where $F_{1}$ and $F_{0}$ are finitely generated free. An $R$-module $M$ is called $w$-coherent if $M$ is of finite type and each finite type submodule of $M$ is of finitely presented type; a ring $R$ is called $w$-coherent if $R$ is $w$-coherent as an $R$-module. Also it is shown that a $\operatorname{ring} R$ is $w$-coherent if and only if every finitely generated ideal of $R$ is of finitely presented type; if and only if every finite type submodule of a free module is of finitely presented type $[20$, Theorem 3.1].

The notion of $w$-flat modules appeared first in [18] when $R$ is a domain and was extended to arbitrary commutative rings in [11]. Recall that an $R$-module $M$ is called a $w$-flat module if the induced map $1 \otimes f: M \bigotimes_{R} A \rightarrow M \otimes_{R} B$ is a $w$-monomorphism for any $w$-monomorphism $f: A \rightarrow B$. Certainly, both flat modules and GV-torsion modules are $w$-flat. Another example of a $w$-flat module is a so-called $w$-projective module. Let $M$ be an $R$-module and set $L(M)=\left(M / \operatorname{tor}_{\mathrm{GV}}(M)\right)_{w}$. Recall from [21] that $M$ is called $w$-projective if $\operatorname{Ext}_{R}^{1}(L(M), N)$ is GV-torsion for every torsionfree $w$-module $N$. Then every $w$-projective module is $w$-flat (see [22, Proposition 2.4]). For easy reference, we list some characterizations of $w$-flat modules. 
Proposition 1.1 (cf. [11, Theorem 3.3]). The following statements are equivalent for an $R$-module $M$.

(1) $M$ is w-flat.

(2) $M_{\mathfrak{m}}$ is a flat $R_{\mathfrak{m}}$-module for all $\mathfrak{m} \in w-\operatorname{Max}(R)$.

(3) $\operatorname{Tor}_{1}^{R}(M, N)$ is $G V$-torsion for all $R$-modules $N$.

(4) $\operatorname{Tor}_{n}^{R}(M, N)$ is $G V$-torsion for all $R$-modules $N$ and all $n \geqslant 1$.

We now introduce the notion of $w$-flat dimension as follows.

Definition. If $M$ is an $R$-module, then $w$-fd $R(M) \leqslant n(w$-fd abbreviates $w$-flat dimension) if there is a $w$-exact sequence of $R$-modules

$$
0 \rightarrow F_{n} \rightarrow \cdots \rightarrow F_{1} \rightarrow F_{0} \rightarrow M \rightarrow 0,
$$

where each $F_{i}$ is a $w$-flat module. The $w$-exact sequence $(\star)$ is called a $w$ flat $w$-resolution of length $n$ of $M$. If no such finite $w$-resolution exists, then $w-\mathrm{fd}_{R}(M)=\infty$; otherwise, define $w-\mathrm{fd}_{R}(M)=n$ if $n$ is the length of a shortest $w$-flat $w$-resolution of $M$.

It is obvious that an $R$-module $M$ is $w$-flat if and only if $w-\operatorname{fd}_{R}(M)=0$, and that $w$ - $\mathrm{fd}_{R}(M) \leqslant \mathrm{fd}_{R}(M)$, where $\mathrm{fd}_{R}(M)$ denotes the classical flat dimension of $M$.

Definition. The $w$-weak global dimension of a ring $R$ is defined by

$$
w \text {-w.gl. } \operatorname{dim}(R)=\sup \left\{w-\mathrm{fd}_{R}(M) \mid M \text { is an } R \text {-module }\right\} \text {. }
$$

Obviously, if we denote the classical weak global dimension of a ring $R$ by w.gl.dim $(R)$, then $w$-w.gl.dim $(R) \leqslant$ w.gl.dim $(R)$. Recall from [20] that a ring $R$ is said to be a $D W$ ring if every ideal of $R$ is a $w$-ideal. Then a ring $R$ is a DW ring if and only if every $R$-module is a $w$-module, if and only if $\operatorname{GV}(R)=\{R\}$ (see [20, Theorem 3.8]). Every ring of weak global dimension at most one is a DW ring. Notice that if $R$ is a DW ring, then $w$-w.gl.dim $(R)=\mathrm{w} \cdot \operatorname{gl} \cdot \operatorname{dim}(R)$.

Let us regard that the $v$-, $t$ - and $w$-operation are well-known star-operations on domains. Recall that an integral domain $R$ is said to be a $P V M D$ if every nonzero finitely generated ideal $I$ is $t$-invertible, that is, there is a fractional ideal $B$ of $R$ such that $(I B)_{t}=R$, equivalently, $(I B)_{w}=R$. It is known that an integral domain $R$ is a PVMD if and only if $R_{\mathfrak{m}}$ is a valuation domain for each $\mathfrak{m} \in w-\operatorname{Max}(R)$. Examples of PVMDs are Prüfer domains, Krull domains, GCD domains, integrally closed coherent domains, etc. In this paper, we prove that an integral domain $R$ is a PVMD if and only if every torsionfree $R$-module is $w$-flat, if and only if $w$-w.gl.dim $(R) \leqslant 1$ (see Theorem 3.5). Let $R[x]$ denote the polynomial ring in one variable $x$ over $R$. Then the well-known Hilbert's Syzygy Theorem states that w.gl.dim $(R[x])=\mathrm{w} \cdot \operatorname{gl} \cdot \operatorname{dim}(R)+1$. However, we show that there is a large class of domains in which the corresponding equality for $w$-weak global dimension does not hold. Namely, it is shown that if $R$ is an integral domain but not a field and if $R[x]$ is $w$-coherent, then $w$-w.gl.dim $(R[x])=$ $w$-w.gl.dim $(R)$ (see Theorem 4.7).

For unexplained terminologies and notations, we refer to $[5,6,16]$. 


\section{On $w$-flat dimension of modules}

We begin with an example of modules of $w$-flat dimension one.

Example 2.1. It is proved in [11, Proposition 3.8] that every GV-torsionfree $w$-flat module over an integral domain $R$ is torsionfree. In fact that is also true for arbitrary commutative rings. Let $a$ be an element of $R$ which is neither a zero-divisor nor a unit. Then $a R \cong R$. By [25, Theorem 2.7], $R / a R$ is a $\mathrm{GV}$-torsionfree $R$-module. Since $R / a R$ is a torsion $R$-module, $R / a R$ is not a $w$-flat module, and so $w$-fd $\mathrm{fd}_{R}(R / a R)=1$.

Before giving characterizations of modules of $w$-flat dimension at most $n$, we prove the following lemma.

Lemma 2.2. Let $N$ be an $R$-module and $0 \rightarrow A \rightarrow F \rightarrow C \rightarrow 0$ a w-exact sequence of $R$-modules with $F$ a $w$-flat module. Then for any $n>0$, the induced map $\operatorname{Tor}_{n+1}^{R}(C, N) \rightarrow \operatorname{Tor}_{n}^{R}(A, N)$ is a w-isomorphism. Hence, $\operatorname{Tor}_{n+1}^{R}(C, N)$ is $G V$-torsion if and only if so is $\operatorname{Tor}_{n}^{R}(A, N)$.

Proof. By [11, Proposition 3.2], there exists a $w$-exact sequence of $R$-modules

$$
\operatorname{Tor}_{n+1}^{R}(F, N) \rightarrow \operatorname{Tor}_{n+1}^{R}(C, N) \rightarrow \operatorname{Tor}_{n}^{R}(A, N) \rightarrow \operatorname{Tor}_{n}^{R}(F, N) .
$$

The result now follows from Proposition 1.1.

Proposition 2.3. The following are equivalent for an $R$-module $M$.

(1) $w-\mathrm{fd}_{R}(M) \leqslant n$.

(2) $\operatorname{Tor}_{n+k}^{R}(M, N)$ is $G V$-torsion for all $R$-modules $N$ and all $k>0$.

(3) $\operatorname{Tor}_{n+1}^{R}(M, N)$ is $G V$-torsion for all $R$-modules $N$.

(4) If $0 \rightarrow F_{n} \rightarrow F_{n-1} \rightarrow \cdots \rightarrow F_{1} \rightarrow F_{0} \rightarrow M \rightarrow 0$ is an exact sequence, where $F_{0}, F_{1}, \ldots, F_{n-1}$ are flat $R$-modules, then $F_{n}$ is $w$-flat.

(5) If $0 \rightarrow F_{n} \rightarrow F_{n-1} \rightarrow \cdots \rightarrow F_{1} \rightarrow F_{0} \rightarrow M \rightarrow 0$ is a w-exact sequence, where $F_{0}, F_{1}, \ldots, F_{n-1}$ are $w$-flat $R$-modules, then $F_{n}$ is $w$-flat.

(6) If $0 \rightarrow F_{n} \rightarrow F_{n-1} \rightarrow \cdots \rightarrow F_{1} \rightarrow F_{0} \rightarrow M \rightarrow 0$ is an exact sequence, where $F_{0}, F_{1}, \ldots, F_{n-1}$ are $w$-flat $R$-modules, then $F_{n}$ is w-flat.

(7) If $0 \rightarrow F_{n} \rightarrow F_{n-1} \rightarrow \cdots \rightarrow F_{1} \rightarrow F_{0} \rightarrow M \rightarrow 0$ is a w-exact sequence, where $F_{0}, F_{1}, \ldots, F_{n-1}$ are flat $R$-modules, then $F_{n}$ is w-flat.

Proof. (1) $\Rightarrow(2)$ We prove (2) by induction on $n \geqslant 0$. For the case $n=0$, $M$ is a $w$-flat module. Then (2) holds by Proposition 1.1. If $n>0$, then there is a $w$-exact sequence $0 \rightarrow F_{n} \rightarrow F_{n-1} \rightarrow \cdots \rightarrow F_{1} \rightarrow F_{0} \rightarrow M \rightarrow 0$, where each $F_{i}$ is a $w$-flat $R$-module. Set $K_{0}=\operatorname{ker}\left(F_{0} \rightarrow M\right)$. Then both $0 \rightarrow K_{0} \rightarrow F_{0} \rightarrow M \rightarrow 0$ and $0 \rightarrow F_{n} \rightarrow F_{n-1} \rightarrow \cdots \rightarrow F_{1} \rightarrow K_{0} \rightarrow 0$ are $w$ exact, and $w-\mathrm{fd}_{R}\left(K_{0}\right) \leqslant n-1$. By induction, $\operatorname{Tor}_{n-1+k}^{R}\left(K_{0}, N\right)$ is GV-torsion for all $R$-modules $N$ and all $k>0$. Thus, it follows from Lemma 2.2 that $\operatorname{Tor}_{n+k}^{R}(M, N)$ is also GV-torsion.

$(2) \Rightarrow(3)$ Trivial. 
$(3) \Rightarrow(4)$ Write $K_{0}=\operatorname{ker}\left(F_{0} \rightarrow M\right)$ and $K_{i}=\operatorname{ker}\left(F_{i} \rightarrow F_{i-1}\right)$, where $i=1, \ldots, n-1$. Then $K_{n-1}=F_{n}$. Since all $F_{0}, F_{1}, \ldots, F_{n-1}$ are flat, $\operatorname{Tor}_{1}^{R}\left(F_{n}, N\right) \cong \operatorname{Tor}_{n+1}^{R}(M, N)$ is GV-torsion for all $R$-modules $N$. Hence, $F_{n}$ is a $w$-flat module by Proposition 1.1.

(4) $\Rightarrow$ (1) Obvious.

$(3) \Rightarrow(5)$ Set $L_{n}=F_{n}$ and $L_{i}=\operatorname{im}\left(F_{i} \rightarrow F_{i-1}\right)$, where $i=1, \ldots, n-1$. Then both $0 \rightarrow L_{i+1} \rightarrow F_{i} \rightarrow L_{i} \rightarrow 0$ and $0 \rightarrow L_{1} \rightarrow F_{0} \rightarrow M \rightarrow 0$ are $w$-exact sequences. By using Lemma 2.2 repeatedly, we will see that $\operatorname{Tor}_{1}^{R}\left(F_{n}, N\right)$ is GV-torsion for all $R$-modules $N$. Thus, $F_{n}$ is a $w$-flat module.

$(5) \Rightarrow(6) \Rightarrow(4),(5) \Rightarrow(7)$, and $(7) \Rightarrow(4)$ are obvious.

Proposition 2.4. Let $M$ be an R-module. Then

(1) $w-\mathrm{fd}_{R}(M) \leqslant n$ if and only if $\mathrm{fd}_{R_{\mathfrak{m}}}\left(M_{\mathfrak{m}}\right) \leqslant n$ for all $\mathfrak{m} \in w-\operatorname{Max}(R)$.

(2) $w-\mathrm{fd}_{R}(M)=\sup \left\{\operatorname{fd}_{R_{\mathfrak{m}}}\left(M_{\mathfrak{m}}\right) \mid \mathfrak{m} \in w-\operatorname{Max}(R)\right\}$.

Proof. Statement (2) follows immediately from (1), and so we only prove (1). Let $0 \rightarrow F_{n} \rightarrow F_{n-1} \rightarrow \cdots \rightarrow F_{1} \rightarrow F_{0} \rightarrow M \rightarrow 0$ be an exact sequence, where $F_{0}, F_{1}, \ldots, F_{n-1}$ are flat $R$-modules. By localizing at $\mathfrak{m} \in w$-Max $(R)$, we get an exact sequence of $R_{\mathfrak{m}}$-modules $0 \rightarrow\left(F_{n}\right)_{\mathfrak{m}} \rightarrow\left(F_{n-1}\right)_{\mathfrak{m}} \rightarrow \cdots \rightarrow\left(F_{1}\right)_{\mathfrak{m}} \rightarrow$ $\left(F_{0}\right)_{\mathfrak{m}} \rightarrow(M)_{\mathfrak{m}} \rightarrow 0$. Also, Proposition 1.1 says that $F_{n}$ is $w$-flat if and only if $\left(F_{n}\right)_{\mathfrak{m}}$ is flat over $R_{\mathfrak{m}}$ for all $\mathfrak{m} \in w-\operatorname{Max}(R)$. Thus the desired conclusion follows.

\section{The $w$-weak global dimension of rings and some characterizations of PVMDs}

In this section, we discuss the $w$-weak global dimension of rings, and then give a characterization of PVMDs. First, we record a consequence of Proposition 2.4 .

Proposition 3.1. For any commutative ring $R$, we have

$$
w \text {-w.gl.dim }(R)=\sup \left\{\text { w.gl.dim }\left(R_{\mathfrak{m}}\right) \mid \mathfrak{m} \in w-\operatorname{Max}(R)\right\} .
$$

Remark 3.2. It is shown in [22, Theorem 4.4] that a commutative ring $R$ is von Neumann regular if and only if every $R$-module is $w$-flat, i.e., $w$-w.gl.dim $(R)=$ 0 . Thus, the $w$-weak global dimension can also measure how far away a ring $R$ is from being von Neumann regular. Moreover, we see that an integral domain $R$ is a field if and only if $w$-w.gl.dim $(R)=0$.

It is worthwhile to point out that the rings of $w$-weak global dimension at most one have been investigated by the first named author and Kim in [22]. In particular, they studied $w$-semihereditary rings. Recall that a ring $R$ is said to be $w$-semihereditary if every finite type ideal of $R$ is $w$-projective; equivalently, every finitely generated ideal of $R$ is $w$-projective. Then a ring $R$ is $w$-semihereditary if and only if $R$ is a $w$-coherent $\operatorname{ring}$ with $w$-w.gl.dim $(R) \leqslant 1$ (see [22, Theorem 4.14]). 
Proposition 3.3. The following statements are equivalent for $R$.

(1) $w$-fd $\mathrm{fd}_{R}(M) \leqslant n$ for all $R$-modules $M$.

(2) $\operatorname{Tor}_{n+k}^{R}(M, N)$ is $G V$-torsion for all $R$-modules $M$ and $N$ and all $k>0$.

(3) $\operatorname{Tor}_{n+1}^{R}(M, N)$ is $G V$-torsion for all $R$-modules $M$ and $N$.

(4) $w-\operatorname{fd}_{R}(R / I) \leqslant n$ for all ideals $I$ of $R$.

(5) $w-\mathrm{fd}_{R}(R / I) \leqslant n$ for all finite type ideals $I$ of $R$.

(6) $w-\mathrm{fd}_{R}(R / I) \leqslant n$ for all finitely generated ideals $I$ of $R$.

(7) $w$-w.gl.dim $(R) \leqslant n$.

Consequently, the w-weak global dimension of $R$ is also determined by the formulas:

$$
\begin{aligned}
w \text {-w.gl.dim }(R) & =\sup \left\{w-\operatorname{fd}_{R}(R / I) \mid I \text { is an ideal of } R\right\} \\
& =\sup \left\{w-\operatorname{fd}_{R}(R / I) \mid I \text { is a finite type ideal of } R\right\} \\
& =\sup \left\{w-\operatorname{fd}_{R}(R / I) \mid I \text { is a finitely generated ideal of } R\right\} .
\end{aligned}
$$

Proof. (1) $\Leftrightarrow(7)$ and $(1) \Rightarrow(4) \Rightarrow(5) \Rightarrow(6)$ are obvious.

$(1) \Rightarrow(2)$ and $(3) \Rightarrow(1)$ are immediate from Proposition 2.3.

(2) $\Rightarrow$ (3) Trivial.

$(6) \Rightarrow(1)$ Let $M$ be an $R$-module and $0 \rightarrow F_{n} \rightarrow F_{n-1} \rightarrow \cdots \rightarrow F_{1} \rightarrow$ $F_{0} \rightarrow M \rightarrow 0$ an exact sequence, where $F_{0}, F_{1}, \ldots, F_{n-1}$ are flat $R$-modules. To complete the proof, it suffices, by Proposition 1.1 , to prove that $\left(F_{n}\right)_{\mathfrak{m}}$ is flat over $R_{\mathfrak{m}}$ for each $\mathfrak{m} \in w-\operatorname{Max}(R)$. Let $A$ be a finitely generated ideal of $R_{\mathfrak{m}}$. Then $A \cong I_{\mathfrak{m}}$ for some finitely generated ideal $I$ of $R$. Thus $w$ - $\mathrm{fd}_{R}(R / I) \leqslant n$ by (6). It follows from Proposition 2.3 that $\operatorname{Tor}_{1}^{R}\left(R / I, F_{n}\right) \cong \operatorname{Tor}_{n+1}^{R}(R / I, M)$ is GV-torsion, and so $\operatorname{Tor}_{1}^{R_{\mathfrak{m}}}\left(R_{\mathfrak{m}} / A,\left(F_{n}\right)_{\mathfrak{m}}\right) \cong \operatorname{Tor}_{1}^{R}\left(R / I, F_{n}\right)_{\mathfrak{m}}=0$. Hence $\left(F_{n}\right)_{\mathfrak{m}}$ is flat over $R_{\mathfrak{m}}$, as desired.

Our next proposition characterizes PVMDs by $w$-projective modules.

Proposition 3.4. The following are equivalent for an integral domain $R$.

(1) $R$ is a PVMD.

(2) Every finitely generated submodule of a projective module is w-projective.

(3) Every finite type submodule of a projective module is w-projective.

(4) Every finitely generated torsionfree module is w-projective.

(5) Every finite type torsionfree module is w-projective.

(6) Every finitely generated submodule of a $w$-projective module is w-projective.

(7) Every finite type submodule of a w-projective module is w-projective.

Proof. (1) $\Leftrightarrow(3) \Leftrightarrow(5)$ See [19].

$(2) \Leftrightarrow(3),(4) \Leftrightarrow(5)$, and $(6) \Leftrightarrow(7)$ follow from [21, Proposition 2.3].

$(6) \Rightarrow(2)$ This is clear.

$(4) \Rightarrow(6)$ Let $N$ be a finitely generated submodule of a $w$-projective $R$ module $M$. Set $M_{1}=M / \operatorname{tor}_{\mathrm{GV}}(M)$ and $N_{1}=N / \operatorname{tor}_{\mathrm{GV}}(N)$. Then the map 
$N_{1} \rightarrow M_{1}$ induced by the inclusion $N \hookrightarrow M$ is a $w$-monomorphism. But $N_{1}$ is a $\mathrm{GV}$-torsionfree $R$-module, so the induced map is a monomorphism. Thus, we may assume that $M$ is GV-torsionfree. By [22, Proposition 2.7], $M$ is a torsionfree module and so is $N$. Hence, $N$ is $w$-projective by (4).

We now offer the promised homological algebra characterization of PVMDs.

Theorem 3.5. The following are equivalent for an integral domain $R$.

(1) $R$ is a PVMD.

(2) Every torsionfree $R$-module is $w$-flat.

(3) $w$-w.gl.dim $(R) \leqslant 1$.

Proof. $(1) \Rightarrow(2)$ Let $M$ be a torsionfree $R$-module and $\mathfrak{m} \in w$-Max $(R)$. Then since $R_{\mathfrak{m}}$ is a valuation domain, $M_{\mathfrak{m}}$ is flat over $R_{\mathfrak{m}}$. Thus, $M$ is $w$-flat by Proposition 1.1.

$(2) \Rightarrow(3)$ Let $M$ be an $R$-module. Then there is an exact sequence of $R$ modules $0 \rightarrow A \rightarrow F \rightarrow M \rightarrow 0$ with $F$ free. Since $A$ is torsionfree, it is $w$-flat by $(2)$. Hence, $w$-fd $R(M) \leqslant 1$, and so $w$-w.gl.dim $(R) \leqslant 1$.

$(3) \Rightarrow(1)$ Let $I$ be a nonzero finitely generated ideal of $R$. As $w$-fd $f_{R}(R / I) \leqslant$ $1, I$ is a $w$-flat ideal. Thus, for any $\mathfrak{m} \in w-\operatorname{Max}(R)$, we have that $I_{\mathfrak{m}}$ is a finitely generated flat ideal of $R_{\mathfrak{m}}$, and so it is projective. Since $R_{\mathfrak{m}}$ is a local domain, $I_{\mathfrak{m}}$ is free. Hence, it follows from [21, Proposition 2.9] that $I$ is a $w$-projective ideal of $R$, and so it is $w$-invertible by [21, Theorem 4.15]. This proves that $R$ is a PVMD.

Now, we provide an example to show that there is a ring of finite $w$-weak global dimension but infinite weak global dimension.

Example 3.6. Let $R$ be the ring $k\left[x_{1}, x_{2}, x_{3}, \ldots\right]$ of polynomials in an infinite number of variables over a field $k$. Then by $[17$, p. 9 , Remark], $R$ is a Krull domain, and so it is a PVMD. By Theorem 3.5, we have $w$-w.gl.dim $(R) \leqslant 1$. But it is known that w.gl.dim $(R)=\infty$.

For the weak global dimension of coherent rings, we have the following result.

Lemma 3.7. If $R$ is a coherent ring, then $\mathrm{w} \cdot \operatorname{gl} \cdot \operatorname{dim}(R) \leqslant n$ if and only if $\mathrm{fd}_{R}(R / \mathfrak{m}) \leqslant n$ for every maximal ideal $\mathfrak{m}$ of $R$.

Proof. Use [6, Corollary 2.5.6, Theorem 2.5.9 and Corollary 2.5.10].

We close this section by giving the $w$-theoretic analogue of Lemma 3.7.

Proposition 3.8. If $R$ is a $w$-coherent ring, then $w$-w.gl.dim $(R) \leqslant n$ if and only if $w-\mathrm{fd}_{R}(R / \mathfrak{m}) \leqslant n$ for each $\mathfrak{m} \in w-\operatorname{Max}(R)$.

Proof. First, we note that $R_{\mathfrak{m}}$ is a coherent local ring for all $\mathfrak{m} \in w$-Max $(R)$. Then $w$-w.gl.dim $(R) \leqslant n$ if and only if w.gl.dim $\left(R_{\mathfrak{m}}\right) \leqslant n$ for all $\mathfrak{m} \in w$-Max $(R)$ (by Proposition 3.1), if and only if $\operatorname{fd}_{R_{\mathfrak{m}}}\left(R_{\mathfrak{m}} / \mathfrak{m} R_{\mathfrak{m}}\right) \leqslant n$ for all $\mathfrak{m} \in w-\operatorname{Max}(R)$ (by Lemma 3.7), if and only if $w-\mathrm{fd}_{R}(R / \mathfrak{m}) \leqslant n$ for all $\mathfrak{m} \in w$-Max $(R)$ (by Proposition 2.4). 


\section{The $w$-weak global dimension of $R[x]$}

In this section, we discuss the $w$-weak global dimension of a polynomial ring $R[x]$ and show that there is a large class of domains without Hilbert's syzygy Theorem for the $w$-weak global dimension. The method used in this section is to utilize $w$-Nagata modules over $w$-Nagata rings.

Let $M$ be an $R$-module. Write

$$
M[x]:=R[x] \bigotimes_{R} M=\left\{\sum_{i} u_{i} x^{i} \mid u_{i} \in M\right\} .
$$

For any $\alpha \in M[x]$, we denote by $c(\alpha)$ the submodule of $M$ generated by the coefficients of $\alpha$ and is called the content of $\alpha$. If $A$ is an $R[x]$-submodule of $M[x]$, then the subset $c(A)$ of all coefficients of elements in $A$ is a submodule of $M$ and is called the content of $A$.

In the following we set

$$
S_{d}=\{f \in R[x] \mid c(f)=R\} \text { and } S_{w}=\left\{f \in R[x] \mid c(f)_{w}=R\right\} .
$$

It is easy to see that $S_{d}$ and $S_{w}$ are multiplicative closed sets of $R[x]$. Note that a finitely generated ideal $J$ of $R$ is a GV-ideal if and only if $J_{w}=R$ (see [25, Proposition 3.5]). From this, we have $S_{w}=\{f \in R[x] \mid c(f) \in \mathrm{GV}(R)\}$. For any $R$-module $M$, we set

$$
R\langle x\rangle:=R[x]_{S_{d}}, \quad M\langle x\rangle:=M[x]_{S_{d}}=R\langle x\rangle \bigotimes_{R} M,
$$

and

$$
R\{x\}:=R[x]_{S_{w}}, \quad M\{x\}:=M[x]_{S_{w}}=R\{x\} \bigotimes_{R} M .
$$

This type of rings was first introduced and studied by Nagata. So we call $R\langle x\rangle$ (resp., $R\{x\}$ ) a Nagata (resp. w-Nagata) ring and $M\langle x\rangle$ (resp., $M\{x\}$ ) a Nagata (resp., w-Nagata) module. We now list some properties of them that will be used in the rest of this section.

Lemma 4.1. (1) $S_{w}=R[x]-\bigcup\{\mathfrak{m}[x] \mid \mathfrak{m} \in w-\operatorname{Max}(R)\}$.

(2) $\{\mathfrak{m}\{x\} \mid \mathfrak{m} \in w-\operatorname{Max}(R)\}$ is the set of all maximal ideals of $R\{x\}$.

(3) Let $M$ be an $R$-module and $\mathfrak{m} \in w-\operatorname{Max}(R)$. Then

$$
M\{x\}_{\mathfrak{m}\{x\}}=M[x]_{\mathfrak{m}[x]}=M_{\mathfrak{m}}\langle x\rangle .
$$

(4) If $M$ is an $R$-module, then $M$ is $w$-flat if and only if $M\{x\}$ is flat over $R\{x\}$.

(5) For any $R$-module $M, w-\mathrm{fd}_{R}(M)=\mathrm{fd}_{R\{x\}}(M\{x\})$.

Proof. For (1) and (2), see [21, Proposition 3.3]. For the proof of (3), see [21, Proposition 3.4]. Also, when $R$ is an integral domain, (4) was proved in [3, Theorem 1.7]. In fact, its proof is true for arbitrary commutative rings. Finally, (5) follows immediately from (4) and [21, Proposition 3.7].

Proposition 4.2. Let $R\{x\}$ be a coherent ring. Then

(1) $R$ is a w-coherent ring.

(2) $w$-w.gl.dim $(R)=$ w.gl.dim $(R\{x\})$. 
Proof. (1) Let $I$ be a finitely generated ideal of $R$. Then $I\{x\}$ is a finitely generated ideal of $R\{x\}$. Since $R\{x\}$ is coherent, $I\{x\}$ is finitely presented, and so $I$ is of finitely presented type by [21, Proposition 3.9]. Thus, $R$ is $w$-coherent.

(2) Let $n$ be a positive integer. Then $w$-w.gl.dim $(R) \leqslant n$ if and only if $w-\operatorname{fd}_{R}(R / \mathfrak{m}) \leqslant n$ for all $\mathfrak{m} \in w-\operatorname{Max}(R)$ (by (1) and Proposition 3.8), if and only if $\operatorname{fd}_{R\{x\}}(R\{x\} / \mathfrak{m}\{x\}) \leqslant n$ for all maximal ideals $\mathfrak{m}\{x\}$ of $R\{x\}$ (by parts (2) and (5) of Lemma 4.1), if and only if w.gl.dim $(R\{x\}) \leqslant n$ (by Lemma 3.7). This proves (2).

In order to exhibit our main theorem, we need to prepare a little.

Lemma 4.3. Let $S$ be a multiplicative closed set of nonzero-divisors of $R$ and let $M$ be a submodule of a free $R$-module. For any $R_{S}$-module $Z$, we denote by $Z_{w}$ the w-envelope of $Z$ as an $R$-module and by $Z_{W}$ the $w$-envelope of $Z$ as a $R_{S}$-module.

(1) If $J \in \mathrm{GV}(R)$, then $J_{S} \in \mathrm{GV}\left(R_{S}\right)$.

(2) $\left(M_{S}\right)_{W}=\left(\left(M_{w}\right)_{S}\right)_{W}$. Consequently, if $R_{S}$ is a $D W$ ring, then $M_{S}=$ $\left(M_{w}\right)_{S}$.

(3) If $M$ is of finite type (resp., of finitely presented type), then $M_{S}$ is a finite type (resp., finitely presented type) $R_{S}$-module. Consequently, if $R_{S}$ is a $D W$ ring, and if $M$ is of finite type (resp., of finitely presented type), then $M_{S}$ is finitely generated (resp., finitely presented) over $R_{S}$.

(4) If $R$ is a w-coherent, then so is $R_{S}$. Consequently, if $R$ is $w$-coherent for which $R_{S}$ is a $D W$ ring, then $R_{S}$ is coherent.

Proof. (1) Since each element of $S$ is not a zero-divisor of $R$, the natural homomorphism $R \rightarrow R_{S}$ is a monomorphism. So we may assume that $R \subseteq R_{S}$. Thus, (1) holds by [24, Lemma 4].

(2) Clearly, $\left(M_{S}\right)_{W} \subseteq\left(\left(M_{w}\right)_{S}\right)_{W}$ as $M \subseteq M_{w}$. On the other hand, let $y \in\left(\left(M_{w}\right)_{S}\right)_{W}$. Then there exists $J \in \mathrm{GV}\left(R_{S}\right)$ such that $J y \subseteq\left(M_{w}\right)_{S}$. Since $J$ is finitely generated, $J=I_{S}$ for some finitely generated ideal $I$ of $R$. Thus, we can find a $c \in S$ with $c I y \subseteq M_{w}$. Also, since $I$ is finitely generated, there is a $J^{\prime} \in \mathrm{GV}(R)$ such that $J^{\prime} I c y \subseteq M$, and so $J_{S}^{\prime} J y \subseteq M_{S}$. By (1), we have $J_{S}^{\prime} \in \mathrm{GV}\left(R_{S}\right)$, and so $J_{S}^{\prime} J \in \mathrm{GV}\left(R_{S}\right)$. Hence, $y \in\left(M_{S}\right)_{W}$.

(3) Let $M$ be a module of finite type. Then $M_{w}=N_{w}$ for some finitely generated submodule $N$ of $M$. By $(2)$, we get $\left(M_{S}\right)_{W}=\left(\left(M_{w}\right)_{S}\right)_{W}=\left(\left(N_{w}\right)_{S}\right)_{W}=$ $\left(N_{S}\right)_{W}$, and so $M_{S}$ is of finite type over $R_{S}$. The case of finitely presented type can be proved similarly to the case of finite type.

(4) Immediate from part (3).

Lemma 4.4. Let $A$ be an ideal of $R[x]$ with $c(A)_{w}=R$. Then $A \cap S_{w} \neq \emptyset$.

Proof. Since $c(A)_{w}=R$, there is, by [25, Proposition 3.7], a finitely generated subideal $B$ of $c(A)$ with $B_{w}=R$. Hence there are finitely many polynomials $f_{1}, \ldots, f_{n} \in A$ with $c\left(f_{1}, \ldots, f_{n}\right)_{w}=R$. For sufficiently large $t_{1}, \ldots, t_{n}$, we 
have that the polynomial $f=x^{t_{1}} f_{1}+\cdots+x^{t_{n}} f_{n}$ satisfies $f \in A$ and $c(f)_{w}=$ $c\left(f_{1}, \ldots, f_{n}\right)_{w}=R$. Thus, $f \in A \cap S_{w}$, as desired.

Following Lucas [12], we use $Q_{0}(R)$ to denote the ring of finite fractions over $R$. Then the ring $Q_{0}(R)$ of finite fractions consists of those elements $\frac{f(x)}{g(x)}$, where $f(x)=\sum_{i=0}^{n} a_{i} x^{i}, g(x)=\sum_{i=0}^{n} b_{i} x^{i} \in R[x]$ and $g(x)$ is a nonzero-divisor of $R[x]$ with the coefficient relations $a_{i} b_{j}=a_{j} b_{i}$ for each $i$ and $j$. For any ideal $I$ of $R$, set $I^{-1}=\left\{z \in Q_{0}(R) \mid z I \subseteq R\right\}$. Recall that an ideal $I$ of $R$ is called semiregular if there is a finitely generated subideal $B$ of $I$ with $\operatorname{ann}(I)=0$. It is proved in [21, Lemma 4.7] that a semiregular finitely generated ideal $I$ is a GV-ideal if and only if $I^{-1}=R$.

Proposition 4.5. For any commutative ring $R, R\{x\}$ is a $D W$ ring.

Proof. Let $A \in \mathrm{GV}(R\{x\})$. Then $A=B_{S_{w}}$ for some finitely generated ideal $B$ of $R[x]$. Set $J=c(B)$. Obviously, $J$ is a finitely generated ideal of $R$. Moreover, we claim that $J$ is semiregular. Indeed, let $J z=0$ for some $z \in R$. Then $A z=0$, and so $z=0$. Also, notice that $B \subseteq J[x]$, and so $A \subseteq J\{x\}$. By [25, Proposition 1.2], we have $J\{x\} \in \operatorname{GV}(R\{x\})$. Let $u \in J^{-1}$. Then $u \in Q_{0}(R)$ and $J u \subseteq R$, and so $J\{x\} u \subseteq R\{x\}$. Hence, $u \in R\{x\}$, and so $u=\frac{f}{g}$, where $f \in R[x]$ and $g \in S_{w}$. As $g u=f$, we get $c(g) u=c(f) \subseteq R$. But $c(g) \in \mathrm{GV}(R)$, so $u \in R$. This proves that $J^{-1}=R$. Thus, $J \in \mathrm{GV}(R)$, i.e., $c(B)_{w}=R$. By Lemma 4.4, $B \cap S_{w} \neq \emptyset$, and so $A=R\{x\}$. It follows that $R\{x\}$ is a DW ring.

Corollary 4.6. If $R[x]$ is a w-coherent ring, then $R\{x\}$ is a coherent ring.

Proof. This follows from Proposition 4.5 and Lemma 4.3(4).

From the above results we get the following theorem.

Theorem 4.7. Let $R$ be an integral domain with quotient field $K$. If $R$ is not a field for which $R[x]$ is w-coherent, then

$$
w \text {-w.gl.dim }(R[x])=w \text {-w.gl.dim }(R) .
$$

Proof. It suffices to show, for a positive integer $n$, that $w$-w.gl.dim $(R[x]) \leqslant n$ if and only if $w$-w.gl.dim $(R) \leqslant n$. Also, by Proposition 4.5 and Corollary 4.6, $R\{x\}$ is a coherent DW ring .

First, suppose that $w$-w.gl.dim $(R[x]) \leqslant n$ and that $M$ is an $R$-module. Let $\mathfrak{m} \in w-\operatorname{Max}(R)$. Then $\mathfrak{m} \neq 0$ as $R$ is not a field. Thus, by using [8, Proposition $1.1]$, it is easy to see that $\mathfrak{m}[x] \in w-\operatorname{Max}(R[x])$. Hence, by Lemma 4.1(3) and Proposition 2.4, we have

$$
\mathrm{fd}_{R\{x\} \mathfrak{m}_{\{x\}}}\left(M\{x\}_{\mathfrak{m}\{x\}}\right)=\mathrm{fd}_{R[x] \mathfrak{m}_{[x]}}\left(M[x]_{\mathfrak{m}[x]}\right) \leqslant n .
$$

It follows that $\operatorname{fd}_{R\{x\}}(M\{x\}) \leqslant n$, and so $w-\mathrm{fd}_{R}(M) \leqslant n$ by Lemma $4.1(5)$. Hence, $w$-w.gl.dim $(R) \leqslant n$. 
Conversely, assume $w$-w.gl.dim $(R) \leqslant n$ and let $P \in w-\operatorname{Max}(R[x])$. If $P \cap R=$ 0 , then $R[x]_{P}=\left(R[x]_{S}\right)_{P_{S}}=K[x]_{P_{S}}$ is a discrete valuation domain, where $S=R-0$. So w.gl.dim $\left(R[x]_{P}\right) \leqslant 1 \leqslant n$. If $P \cap R \neq 0$, then by [8, Proposition 1.1], $\mathfrak{m}:=P \cap R \in w-\operatorname{Max}(R)$ and $P=\mathfrak{m}[x]$. It follows from Lemma 4.1(3) and Proposition 4.2(2) that

$$
\text { w.gl.dim }\left(R[x]_{P}\right)=\text { w.gl.dim }\left(R\{x\}_{\mathfrak{m}\{x\}}\right) \leqslant \text { w.gl.dim }(R\{x\}) \leqslant n .
$$

Thus, $w$-w.gl.dim $(R[x]) \leqslant n$ by Proposition 3.1 .

Example 4.8. Notice that an integral domain $R$ is a PVMD if and only if so is $R[x]$ (see [9, Theorem 3.7]). Also, from Proposition 3.4 and [21, Theorem 2.19], it is easy to see that every PVMD is a $w$-coherent domain. Thus, every PVMD which is not a field satisfies the condition of Theorem 4.7. However, the converse is not true in general. In fact, every Noetherian domain which is not integrally closed satisfies the condition of Theorem 4.7 but not a PVMD.

Acknowledgement. The authors would like to thank the referee for his/her careful reading of the manuscript and several useful comments. This work was partially supported by NSFC (No. 11171240) and the Specialized Research Fund for the Doctoral Program of Higher Education (No. 20125134110002).

\section{References}

[1] G. W. Chang, Prüfer *-multiplication domains, Nagata rings, and Kronecker function rings, J. Algebra 319 (2008), no. 1, 309-319.

[2] - On characterizations of Prüfer v-multiplication domains, Korean J. Math. 18 (2010), no. 4, 335-342.

[3] G. W. Chang, H. Kim, and J. W. Lim, Integral domains in which every nonzero t-locally principal ideal is t-invertible, Comm. Algebra 41 (2013), no. 10, 3805-3819.

[4] S. El Baghdadi and S. Gabelli, Ring-theoretic properties of PvMDs, Comm. Algebra 35 (2007), no. 5, 1607-1625.

[5] R. Gilmer, Multiplicative Ideal Theory, Pure and Applied Mathematics, No. 12. Marcel Dekker, Inc., New York, 1972.

[6] S. Glaz, Commutative Coherent Rings, Lecture Notes in Mathematics, 1371. SpringerVerlag, Berlin, 1989

[7] M. Griffin, Some results on v-multiplication rings, Canad. J. Math. 19 (1967), 710-722.

[8] E. Houston and M. Zafrullah, On t-invertibility. II, Comm. Algebra 17 (1989), no. 8, 1955-1969.

[9] B. G. Kang, Prüfer v-multiplication domains and the ring $R[X]_{N_{v}}$, J. Algebra 123 (1989), no. 1, 151-170.

[10] Some questions about Prüfer v-multiplication domains, Comm. Algebra 17 (1989), no. 3, 553-564.

[11] H. Kim and F. Wang, On LCM-stable modules, J. Algebra Appl. 13 (2014), no. 4, 1350133, 18 pp.

[12] T. G. Lucas, Strong Prüfer rings and the ring of finite fractions, J. Pure Appl. Algebra 84 (1993), no. 1, 59-71.

[13] R. Matsuda, Notes of Prüfer v-multiplication rings, Bull. Fac. Sci. Ibaraki Univ. Ser. A No. 12 (1980), 9-15.

[14] J. L. Mott and M. Zafrullah, On Prüfer v-multiplication domains, Manuscripta Math. 35 (1981), no. 1-2, 1-26. 
[15] G. Picozza, A note on Prüfer semistar multiplication domains, J. Korean Math. Soc. 46 (2009), no. 6, 1179-1192.

[16] J. J. Rotman, An Introduction to Homological Algebra, Pure and Applied Mathematics, 85. Academic Press, Inc. [Harcourt Brace Jovanovich, Publishers], New York-London, 1979.

[17] P. Samuel, Lectures on unique factorization domains, Notes by M. Pavman Murthy, Tata Institute of Fundamental Research Lectures on Mathematics, No. 30. Tata Institute of Fundamental Research, Bombay, 1964.

[18] F. Wang, On w-projective modules and w-flat modules, Algebra Colloq. 4 (1997), no. 1, $111-120$

[19] - w-modules over a PVMD, Proc. ISTAEM, Hong Kong, 117-120, 2001

[20] Finitely presented type modules and $w$-coherent rings, J. Sichuan Normal Univ. 33 (2010), 1-9.

[21] F. Wang and H. Kim, Two generalizations of projective modules and their applications, J. Pure Appl. Algebra 219 (2015), no. 6, 2099-2123.

$[22] \_, w$-injective modules and $w$-semi-hereditary rings, J. Korean Math. Soc. 51 (2014), no. 3, 509-525.

[23] F. Wang and J. Zhang, Injective modules over $w$-Noetherian rings, Acta Math. Sinica (Chin. Ser.) $\mathbf{5 3}$ (2010), no. 6, 1119-1130.

[24] H. Yin and Y. Chen, w-overrings of $w$-Noetherian rings, Studia Sci. Math. Hungar. 49 (2012), no. 2, 200-205.

[25] H. Yin, F. Wang, X. Zhu, and Y. Chen, w-modules over commutative rings, J. Korean Math. Soc. 48 (2011), no. 1, 207-222.

[26] S. Zhao, F. Wang, and H. Chen, Flat modules over a commutative ring are $w$-modules, J. Sichuan Normal Univ. 35 (2012), 364-366.

FANGGUI WANG

College of Mathematics and Software Science

Sichuan Normal University

Chengdu, Sichuan 610068, P. R. China

E-mail address: wangfg2004@163.com

LEI QIAO

College of Mathematics and Software Science

Sichuan Normal University

Chengdu, Sichuan 610068, P. R. China

E-mail address: qiaolei5@yeah.net 